\title{
Algunas perspectivas investigativas internacionales contemporáneas sobre formación y pedagogía de los derechos humanos
}

\author{
Elliot Parra Ávila ${ }^{1}$ \\ Universidad Militar Nueva Granada - Colombia \\ Recibido: 14/06/2019 - Aprobado:23/11/2019 \\ DOI: https://doi.org/10.22490/26655489.3214
}

\section{Resumen}

La pedagogía en derechos humanos cobra cada vez mayor fuerza; en especial en el área de la investigación de programas de posgrado. El objetivo de este texto es ilustrar el estado actual de las investigaciones sobre el campo de la formación y la pedagogía de los derechos humanos desde una perspectiva internacional. En vista de la escasa documentación que exponga sus campos de estudio, se han identificado tres:

a. Estudios relacionados con derechos humanos y cultura.

b. Enfoques diferenciales sobre sujetos de derechos humanos.

c. Estudios de caso sobre países en particular. La investigación internacional en derechos humanos es amplia y diversa, supera las visiones clásicas de los conflictos armados y expone nuevas experiencias que pueden emularse nacionalmente.

Palabras clave: pedagogía de los derechos humanos, didáctica de los derechos humanos, enseñanza de los derechos humanos, investigación en derechos humanos.

1 Abogado y Magíster en Derecho de la Universidad Nacional de Colombia, Especialista en Derecho Procesal de la Universidad del Rosario y Master of Research del European University Institute. Docente Asociado de la Universidad Militar Nueva Granada y profesor universitario de pregrado y posgrado, par académico de Conaces y Conjuez del Tribunal Administrativo de Cundinamarca. ORCID: https://orcid.org/0000-0001-63767012. correos electrónicos: elliotparra@gmail.com y elliot.parra@unimilitar.edu.co 


\section{Introducción}

La experiencia cotidiana indica que muchos estudiantes de posgrado se abstienen de elaborar investigaciones sobre los temas pedagógicos en derechos humanos bajo el supuesto de que no es un campo con suficiente información para elaborar estados del arte y marcos teóricos. Aunque la situación descrita puede ser cierta en el caso colombiano, el trabajo demostrará que el debate en el plano internacional se encuentra activo y en plena expansión.

El objetivo de este texto es ilustrar el estado de las investigaciones sobre la formación y la pedagogía de los derechos humanos desde una perspectiva internacional; esto es, estudios realizados fuera de Colombia o realizados sobre casos distintos al colombiano, tal como se expuso inicialmente en el artículo "Temas y tendencias internacionales contemporáneas en investigación sobre pedagogía de los derechos humanos" (2016). El presente artículo lo complementa con la exposición de tres perspectivas adicionales.

\section{Metodología}

Por ser la fuente con denominación más aproximada al tema de estudio, en primer lugar, se acudió a la Base de datos sobre la educación y la capacitación en Derechos Humanos de la Oficina del Alto Comisionado de las Naciones Unidas (consolidada desde 2005) y en ella se buscó bajo los términos "pedagogía" y "pedagogy". Solo se obtuvieron seis resultados².

Como el criterio "contemporáneo" es entendido en el trabajo como trabajos que correspondieran a las últimas dos décadas y que describieran experiencias significativas en las diversas áreas de trabajo de la formación y pedagogía en derechos humanos, de las cinco fuentes halladas solo las dos de 2018 se justan a nuestros criterios.

2 Empowering visions: toward a dialectical pedagogy of human rights (Ely-Yamin, 1993), Pedagogy of the oppressed (Freire, 2000), Human rights education social change and human values: Asia-Pacific Regional Conference on Education for Human Rights 3-6 February 1999 (UNESCO Chair in Peace, Human Rights and Democracy India, 2000), Proyecto de Centro de recursos pedagógicos en derechos humanos de Aiete (Gobierno Vasco. Secretária General de Derechos Humanos, 2018) y Pedagogy of human rights education and secondary school pre-service teachers in India: philosophy and praxis (Anamika, 2018) 
Por ello, en segundo lugar, se acudió a la base de datos ProQuest. La elección de esta base de datos se fundamenta en el trabajo de Gusenbauer (2019), quien muestra cómo ProQuest es la más grande base de datos con textos completos disponibles, aunque no es la más grande en términos absolutos (lo es Google Scholar). La búsqueda en ProQuest ${ }^{3}$ arrojó 203 resultados, que fueron agrupados por la mayor frecuencia de palabras claves.

Dicha agrupación arrojó seis grandes ámbitos contemporáneos de la pedagogía de los derechos humanos, tres de los cuales ya han sido expuestos (Parra, 2016): discusiones pedagógicas sobre los derechos humanos, investigaciones sobre la didáctica de los derechos humanos y propuestas curriculares en derechos humanos. Este texto versará sobre los tres ámbitos restantes: 1) Estudios relacionados con derechos humanos y cultura, 2) Enfoques diferenciales sobre sujetos de derechos humanos y 3) Estudios de caso sobre países en particular.

\section{Derechos humanos y cultura}

Preliminarmente se debe mencionar la aspiración ecuménica de la Campaña Global para la Educación para la Paz (GCPE por sus siglas en inglés) que tuvo orígenes en los años 90 y pretende un cambio cultural mundial que concientice a los pueblos sobre el valor de la paz sobre la guerra (Reardon, 2002).

Una interesante interrelación entre problemas curriculares y estudios con enfoque diferencial que representan patrones culturales puede ser el estudio de Vithal (2005). El caso surafricano se estudió en clave de género dentro de las clases de matemáticas, donde el nuevo currículo pide a los docentes que apliquen en sus salones de clase una pedagogía más práctica, orientada a la actividad y conectada con la vida de sus alumnos. Basándose en los datos de un estudio más amplio que explora las relaciones teoría-práctica en la educación matemática, este trabajo muestra cómo este tipo de prácticas progresistas, respecto a la enseñanza de la medición de áreas que exige a los alumnos utilizar diferentes instrumentos de medición en los terrenos de su propia escuela para aprender sobre longitud y perímetro, mostraron que el aprendizaje estaba profundamente determinado por el género. En dos contextos diferentes, una escuela de un

3 Términos "human rights" y "pedagogy" sin "Colombia" en cualquier campo excepto texto completo, entre 2002 y 2018. 
municipio "africano" y una de uno predominantemente "indio", las niñas de la misma clase de matemáticas de sexto grado enfrentaban directamente el sexismo mientras luchaban por tener la oportunidad de participar en la actividad y aprender a medir (que es una importante competencia matemática). El artículo analizó los datos con referencia a los requisitos sobre derechos humanos de los nuevos enfoques y planes de estudio nacionales para hacer frente a las desventajas y la discriminación de las niñas en las clases de matemáticas.

\section{$\underline{\text { Religión }}$}

Los riesgos (la "doble cara de Jano") de las religiones en el mundo contemporáneo, con su potencial de ser fuerzas positivas, pero también negativas, son estudiados por Gundara (2000). Los educadores pueden utilizar los aspectos y dimensiones positivas de la religión para fomentar la comprensión entre religiones y culturas. En este sentido, los educadores pueden utilizar los instrumentos de derechos humanos que la mayoría de las naciones han aprobado para asegurarse de que la base jurídica y laica del Estado proporcione seguridad y protección a todas las religiones. El artículo analiza estos temas y el papel de la educación intercultural como facilitador de la educación y la comprensión entre religiones.

El tema de la religión también es abordado, en perspectiva interdisciplinaria, por Gearon (2008) quien, tomando el totalitarismo como núcleo conceptual, comienza por definir algunos de los parámetros históricos y políticos del liberalismo moderado en sentido amplio, y los extremos de la represión y el control totalitario. El artículo aplica y desarrolla este análisis a la educación religiosa, espiritual y de los derechos humanos. Aunque el texto maneja un nivel simbólico filosófico para su discusión y posterior aplicación pedagógica en planes de estudio, mediante el uso de la forma literaria de la novela representa un ejemplo de cómo el totalitarismo ha sido retratado y resistido, y una advertencia implícita sobre los peligros del exceso de politización, ya sea la religiosa o espiritual.

La secularidad de la educación y su relación tiene expresión en tres estudios. Primero, el trabajo de Print y otros (Print, Ugarte, Naval, \& Mihr, 2008) sobre educación moral. Al hacer un 
balance del Decenio de las Naciones Unidas para la Educación en Derechos Humanos (1995-2004), se revisó críticamente la evolución de la educación en derechos humanos (en adelante HRE por sus siglas en inglés) durante esos diez años en el contexto de la educación moral. Se argumentó que, a pesar de algunos éxitos modestos, la década careció de dirección y de un gran impacto y no pudo preparar una base sólida para lograr alcanzar la HRE internacionalmente. Estos resultados explican en gran parte la decisión de las Naciones Unidas (ONU) en 2005 para iniciar el Programa Mundial para la Educación en Derechos Humanos. El balance del autor es que en general, la contribución de la ONU a la construcción de la HRE y la educación moral ha sido, como mucho, un éxito marginal debido en gran medida a las debilidades inherentes de la organización, así como la incapacidad de la ONU para influir en los estados miembros.

En segundo lugar, está Jawoniyi (2011) quien exploró la cuestión de si y cómo la religión se debe enseñar en las escuelas financiadas por el Estado en las sociedades seculares, liberales, democráticos, pluralistas y posmodernas. Las dos variables que se deben considerar son la sostenida y dominante presencia de la religión en la esfera pública y la necesidad de que los niños tengan profundo y claro conocimiento y comprensión críticos del papel de la religión en el mundo actual. De la misma manera, es necesario proteger a los niños de ser sometidos a un adoctrinamiento a través de programas de educación religiosa que se ofrecen en las escuelas, riesgo que no se puede subestimar. Usando marcos internacionales de derechos humanos y la jurisprudencia, pertinentes para evaluar los diferentes enfoques pedagógicos de la educación religiosa, Jawoniyi sostiene que una educación religiosa multirreligiosa y no confesional -dado su enfoque transmitir conocimientos a los niños de una manera objetiva, crítica y pluralista, sin adoctrinamiento- es el modelo pedagógico que mejor se ajusta a los principios del derecho internacional de los derechos humanos.

El tercer estudio es el de Bowie (2012) sobre secularismo. El autor se cuestiona sobre si la HRE está respondiendo adecuadamente al giro post secular en la reflexión sobre el lugar y la naturaleza de la religión en la sociedad. Utiliza dicho giro para describir el descrédito de la teoría de la secularización, reconocimiento de la religión como una fuerza cultural global duradera y generalizada, y la emergencia resultante de un discurso 
que reconoce diferentes secularidades y múltiples modernidades. Este artículo caracteriza el giro post secular, señala el discurso emergente e identifica la falta de una respuesta adecuada de la HRE inglesa, que el autor considera comprometida con una teoría cada vez más obsoleta de la secularización.

\section{Ciudadanía}

Existen posturas en contra de la tendencia de la educación en derechos humanos, donde se enseñan los derechos humanos en forma de educación para la ciudadanía En opinión de Hung (2012), la educación para la ciudadanía y la educación en derechos humanos no pueden ser tomados como reemplazable la una por la otra. Sustentar la idea de la ciudadanía implica distinguir entre personas "políticamente calificadas" y "políticamente no calificadas". Esta distinción implica una violación de los derechos humanos en nombre de la solidaridad social y la seguridad. Se propone que la educación ciudadana podría implicar discriminación / exclusión a pesar de que dice promover la solidaridad y los derechos humanos. Por otra parte, tener derechos no depende de la ciudadanía, sino simplemente de la propia vida humana, lo que tiene dos implicaciones educativas. En primer lugar, los derechos humanos y la educación para la ciudadanía no pueden verse como equivalentes. En segundo lugar, los educadores deben estar atentos a los peligros de que la educación ciudadana implique una posible exclusión.

Desde una visión menos escéptica, Starkey (2012) discute sobre el mismo tema, dado que la educación para la ciudadanía, que se define como aprender a vivir juntos, requiere un acuerdo sobre ciertos principios comunes. Un propósito central de un sistema estatal de educación es la transmisión de los estándares normativos comunes, tales como los derechos humanos y las libertades fundamentales que sustentan las sociedades democráticas liberales. El documento identifica las raíces conceptuales de la Declaración Universal de los Derechos Humanos (DUDH) en el concepto sociológico de la utopía y la ilustración cosmopolita. En la Declaración Universal, la visión de las libertades que pueden promover la prosperidad humana proporciona una manera precisa de conceptualizar los límites al poder del Estado. Mientras que los derechos humanos no es una teoría general, el concepto tiene la propiedad enormemente 
beneficiosa de permitir que las personas cuyos sistemas de valores son diversos y aparentemente incompatibles, reconozcan y acepten las normas y principios comunes que hacen posible la vida en sociedad. Las implicaciones de esto son que la educación en derechos humanos es legítimamente reconocida como un componente esencial de la educación ciudadana.

Pero como lo advirtió Hung, la educación para la ciudadanía tiene sus riesgos, y uno de ellos es el patriotismo. Zembylas (2013) examina las tensiones morales, políticas y pedagógicas que se crean por la confusión entre patriotismo y derechos humanos, y esboza una respuesta a estas tensiones en el contexto de la educación crítica. El artículo comienza con una breve revisión de las distintas formas de patriotismo, especialmente en lo que se refiere a los derechos humanos, y explica por qué algunas de estas formas pueden ser moral o políticamente valiosas. Luego ofrece una breve descripción de las críticas de los derechos humanos, sobre todo desde la perspectiva de Foucault, la escuela crítica y teoría postcolonial, y hace hincapié en que las perspectivas fundamentalistas de los derechos humanos deben ser refutadas constantemente. La siguiente parte del artículo discute la manera de superar los problemas de incompatibilidad entre el patriotismo y los derechos humanos. La última parte propone que un "acercamiento" entre el patriotismo y los derechos humanos en el contexto de la educación crítica debe tener en cuenta que los sentimientos patrióticos (como una forma de amor por la patria) constituye una forma particular de "educación emocional". Como tal, la enseñanza tanto del patriotismo como de los derechos humanos se beneficiaría de la noción de "pedagogías críticas de la emoción" que cuestionan los compromisos emocionales de patriotismo y de los derechos humanos y las consecuencias de estos compromisos.

\section{Enfoques diferenciales}

Hasta acá hemos visto estudios que se enfocan en el aula y en la formación escolar de derechos humanos, pero como lo señala Andreopolus (2002), aunque la HRE tiene una larga historia, sólo recientemente hemos comenzado a darnos cuenta de su potencial transformador. Un catalizador crítico en este proceso es el ethos global emergente de la "rendición de cuentas". Dentro de este contexto, la HRE puede desempeñar un 
papel crucial en la construcción de una cultura universal de los derechos humanos, inspirada en un compromiso comprometido con la creencia de que los seres humanos tienen la capacidad y la responsabilidad de hacer la diferencia. En ese esfuerzo, la formación de profesionales es vital, especialmente de aquellos profesionales que pueden influir en el debate público sobre la rendición de cuentas y la gobernabilidad. Las iniciativas innovadoras de HRE, que se caracterizan por un compromiso de aprendizaje experiencial y de cooperación internacional y regional entre los actores estatales y no estatales, son para el autor un paso en la dirección correcta.

Para garantizar que los derechos de los niños se hagan realidad, Hart (2002) propone que las estrategias se enfoquen en "escuchar su voz". Una de esas estrategias es la pedagógica, ya que se ha demostrado que los educadores que buscan las opiniones de los estudiantes, que promueven el aprendizaje que se ocupa de cuestiones de importancia para su vida cotidiana, que eliminan el miedo a la humillación y la falta de manifestación de pensamiento divergente, y que muestren respeto por las opiniones de todos los niños y adultos en la escuela, fortalecen la confianza de los niños a expresar sus opiniones. Las escuelas deberían implementar prácticas basadas en la teoría del constructivismo, que incorporen la escuela y el servicio comunitario y que establezcan una influencia sustancial de los estudiantes en la toma de decisiones sobre temas importantes, para mostrar respeto y apoyo a tendencia de sus niños a expresar sus opiniones y a participar en la traducción de esas opiniones en acciones constructivas.

Otra faceta de la problemática de los derechos humanos es la del trabajo infantil. El breve artículo de Liebel (2002) ilustra las relaciones que existen entre educación y trabajo infantil, que no se condena a priori, sino que se considera como un hecho ambivalente: puede obstaculizar o puede apoyar la práctica de los derechos de los niños, dependiendo tanto de las condiciones de trabajo y de si se encuentran en un contexto sociocultural donde sean respetados ellos y su trabajo y donde tengan la oportunidad de unirse en defensa de sus intereses. La acción educativa puede hacer una importante contribución a la observancia de los derechos de los niños, ya que crea o apoya un ambiente en el que los niños que trabajan encuentran la comprensión de sus 
preocupaciones y puntos de vista y fortalecen su autoconfianza. La acción educativa puede incluir el apoyo en situaciones de conflicto, la mejora de las condiciones de trabajo, y la creación de patrones de trabajo alternativos en los que los niños pueden cooperar y actuar autónomamente.

El grupo específico de los niños soldados es estudiado por Druba (2002), quien propone intervenciones pedagógicas para reducir el número de los niños soldados. Dichas intervenciones se pueden dar principalmente a la educación primaria, pero también en la formación profesional, los servicios sociales y los nuevos conceptos de educación para la reconstrucción y la educación para la reconciliación. El texto recoge los relatos de experiencias y propuestas de programas de rehabilitación y reinserción ya publicados, como los de Jareg y McCallin (1993), McCallin (1998) y el propio Druba (2000), recordando que, si bien la educación sí importa, por sí sola no puede prevenir el surgimiento de niños soldados. La conclusión, un poco pesimista, es que sólo es posible reducir la participación de los niños en la guerra si los líderes políticos están más interesados en el bienestar de los niños que en las estrategias militares.

La investigación de las perspectivas históricas en materia de derechos humanos y los derechos del niño, es el objeto del texto de Burke (2007). Reconociendo que la importancia de los derechos universales de los niños es fundamental en un mundo diferenciado y pluralista, que, gracias al aumento de la interdependencia económica mundial y sus cambios consiguientes, requeriría una amplia gama talentos para mantener la paz y la cooperación. El autor propone un análisis de los derechos del niño considerándolos tanto derechos humanos positivos como negativos que, en el caso del contexto del derecho a la educación, deben incluir prácticas de enseñanza que respeten las capacidades de desarrollo, cognitivas e intelectuales de los niños.

El derecho internacional humanitario también tiene cabida en los estudios pedagógicos liderados por Day (Day, Vandiver, \& Janikowski, 2003). Para ellos, la rápida expansión del derecho internacional y el establecimiento de una Corte Penal Internacional han dado lugar a nuevas instituciones de justicia que no deben pasarse por alto en el plan de estudios de la justicia penal. El genocidio como el punto focal de todo un curso o una unidad dentro de otros cursos, proporciona a los instructores un marco 
organizativo para hacer frente a una amplia variedad de temas relacionados con el derecho internacional. En el trabajo se analiza cómo el delito de genocidio se puede integrar en el currículo de justicia penal y de las oportunidades que ofrece para examinar el derecho internacional, la historia, los acontecimientos actuales y la interdependencia de los componentes del sistema de justicia penal. Contiene la descripción y evaluación de un curso sobre el genocidio para los últimos años de pregrado y primeros de posgrado.

En el mismo ámbito del derecho penal internacional, el estudio de Ross y Rothe (2007) sobre los delitos estatales señala que, durante las últimas dos décadas, un número cada vez mayor de criminólogos han llevado a cabo investigaciones rigurosas sobre Crímenes de Estado y han tratado de difundirlas ampliamente mediante el desarrollo de cursos que abordan específicamente dichos delitos. Sin embargo, la enseñanza de este tema, al igual que otras cuestiones polémicas, no es tan sencilla como algunos podrían esperar. Los autores presentan un marco para entender la enseñanza de los crímenes de Estado en los programas de pregrado y posgrado en universidades de Estados Unidos. Con el fin de transmitir la experiencia actual, los autores entrevistaron docentes que imparten cursos sobre la materia, y con base en los resultados, concluyen que las exigencias de los currículos de criminología y de justicia penal, el enfoque en los delitos comunes callejeros y el enfoque de los estudiantes en general orientado al litigio, impiden la adopción a gran escala de clases sobre crímenes de Estado y han servido para marginalizar el tema en las universidades estadounidenses.

Las relaciones entre metodologías y posguerra son el objeto de la investigación de Austin y otros (Austin, McDowell, \& Sacko, 2006). En su artículo se describe una simulación para estudiantes de pregrado de la Academia de la Fuerza Aérea de los Estados Unidos que formulaba diferentes regímenes para Irak tras el derrocamiento del régimen de Saddam Hussein. Este ejercicio refuerza la comprensión y el conocimiento de los estudiantes en una amplia gama de temas, incluyendo jurídicos y políticos, la toma de decisiones en grupo, el derecho internacional, la diplomacia y los derechos humanos mediante la participación activa de los estudiantes en la formulación de políticas y en negociaciones entre grupos. Los estudiantes diseñan el aparato jurídico principal y luego se unen para ejecutar la simulación. Los cuatro componentes clave de los enfoques 
La investigación de las perspectivas históricas en materia de derechos humanos y los derechos del niño, es el objeto del texto de Burke (2007). Reconociendo que la importancia de los derechos universales de los niños es fundamental en un mundo diferenciado y pluralista, que, gracias al aumento de la interdependencia económica mundial y sus cambios consiguientes, requeriría una amplia gama talentos para mantener la paz y la cooperación. El autor propone un análisis de los derechos del niño considerándolos tanto derechos humanos positivos como negativos que, en el caso del contexto del derecho a la educación, deben incluir prácticas de enseñanza que respeten las capacidades de desarrollo, cognitivas e intelectuales de los niños.

El derecho internacional humanitario también tiene cabida en los estudios pedagógicos liderados por Day (Day, Vandiver, \& Janikowski, 2003). Para ellos, la rápida expansión del derecho internacional y el establecimiento de una Corte Penal Internacional han dado lugar a nuevas instituciones de justicia que no deben pasarse por alto en el plan de estudios de la justicia penal. El genocidio como el punto focal de todo un curso o una unidad dentro de otros cursos, proporciona a los instructores un marco organizativo para hacer frente a una amplia variedad de temas relacionados con el derecho internacional. En el trabajo se analiza cómo el delito de genocidio se puede integrar en el currículo de justicia penal y de las oportunidades que ofrece para examinar el derecho internacional, la historia, los acontecimientos actuales y la interdependencia de los componentes del sistema de justicia penal. Contiene la descripción y evaluación de un curso sobre el genocidio para los últimos años de pregrado y primeros de posgrado.

En el mismo ámbito del derecho penal internacional, el estudio de Ross y Rothe (2007) sobre los delitos estatales señala que, durante las últimas dos décadas, un número cada vez mayor de criminólogos han llevado a cabo investigaciones rigurosas sobre Crímenes de Estado y han tratado de difundirlas ampliamente mediante el desarrollo de cursos que abordan específicamente dichos delitos. Sin embargo, la enseñanza de este tema, al igual que otras cuestiones polémicas, no es tan sencilla como algunos podrían esperar. Los autores presentan un marco para entender la enseñanza de los crímenes de Estado 
educativos de aprendizaje activo: objetivos, parámetros de diseño, procedimientos y evaluación y debate, proporcionan una comprensión completa de los objetivos, la configuración, el proceso y las ventajas de las sinergias a través del plan de estudios para el problema de la justicia iraquí de posguerra.

Otra experiencia en educación de militares es la que narran Bengs y Augustin (2010) en su trabajo sobre el origen y el alcance de la obligación legal internacional para educar a los militares en el derecho de los conflictos armados (DICA ${ }^{4}$ ). Los sistemas de educación de la Fuerza Aérea de los Estados Unidos para sus candidatos a oficial sirven como herramienta para identificar los requisitos básicos cuantitativos y cualitativos en educación en DICA y para evaluar cómo esos requerimientos básicos se alcanzan por parte de los oficiales de instrucción de la Fuerza Aérea. El artículo sostiene que los requerimientos básicos ya no son suficientes para los militares profesionales que participan

en conflictos armados modernos. La metodología cuantitativa y cualitativa empleada por la Academia de la Fuerza Aérea de los Estados Unidos para lograr un nivel ambicioso de educación en DICA se ofrece como ejemplo de lo que deberían ser los nuevos requerimientos en educación en DICA, dada la naturaleza única de los conflictos armados modernos.

\section{Estudios de casos geográficos}

\section{$\underline{\text { Países musulmanes }}$}

El caso árabe en general es tratado por Baccouche (2002) en un artículo que describe un acercamiento informal a la educación en derechos humanos, anclada en la realidad y con base en la experiencia adquirida en toda la región árabe y, más particularmente, en el Instituto Árabe de Derechos Humanos. Se defiende que el enfoque del Instituto se podría adoptar en mayor escala por parte de las organizaciones que trabajan en este ámbito, no sólo en la región árabe, sino también en otras regiones del mundo. El artículo pone de relieve el papel fundamental de

4 Es importante anotar que la denominación DICA para el Derecho Internacional de los Conflictos Armados es típicamente latinoamericana, en especial argentina, por eso es curioso que un texto anglosajón hable en términos de "law of armed conflict" y que use la sigla LOAC. 
los gobiernos y de la importancia de la cooperación entre los niveles gubernamentales y no gubernamentales con el fin de implementar este tipo de educación en derechos humanos en situaciones de aprendizaje informal, y por lo tanto para difundir una cultura universal de los derechos humanos.

La particular realidad de Kuwait es ilustrada por Al-Nakib (2012), quien señala acertadamente (como se verá más abajo en el caso africano) que mientras que los marcos de derechos humanos universales y modelos democráticos de gobierno han ganado el apoyo y adhesión mundiales, a menudo entran en tensión con las prácticas culturales y religiosas locales. En Kuwait surgen tensiones entre su constitución, el sistema legal y el islam con la consecuencia de hallarse varios grupos marginados. Estas tensiones se extienden al sistema educativo. El trabajo exploró tanto teórica como contextualmente el potencial de un módulo curricular introducido en 2006 en la profundización de la educación para la ciudadanía democrática en Kuwait. Un análisis teórico reveló que el módulo, introducido sin alterar el espíritu autoritario del sistema, representa un compromiso débil con los conceptos de derechos y de democracia. Sin embargo, su promulgación dentro de la escuela estudiada -que representa una escuela estatal autoritaria y a la vez miembro de la Red del Plan de Escuelas Asociadas de la UNESCO (ASPnet por sus siglas en inglés)- descubrió su potencial más profundo y quizás descubrió la razón de su rápido descenso de un programa de tres años a uno de un año.

La experiencia indonesia se puede leer en el texto de Inagaki (2002), quien explica que el proyecto de educación en derechos humanos busca mejorar la comprensión de las cuestiones de derechos humanos de los docentes y que para familiarizarlos con la práctica docente, se realizaron talleres de capacitación sobre educación en derechos humanos llevados a cabo por la Facultad de Derecho y la Facultad de Ciencias Socio-Políticas del Instituto de Enseñanza y Ciencias de la Educación en Kupang y la Comisión Nacional de Indonesia para los Derechos Humanos. El enfoque de la enseñanza de la educación en derechos humanos en las escuelas piloto ha estado en la promoción de valores que afirmen los derechos humanos, a través de actividades extracurriculares, la interacción diaria en el aula y la integración de la educación en derechos humanos en temas relevantes como las ciencias sociales y la educación cívica. 
Reconocido como un país musulmán caracterizado por un fuerte laicismo, el caso de Turquía es expuesto por Cayir \& Bağlı (2011). En 1998 se incorporaron obligatoriamente cursos sobre derechos humanos en el plan de estudios de las escuelas secundarias, constituyendo un primer paso importante en el desarrollo del respeto por los derechos humanos y las responsabilidades entre la generación más joven en Turquía. Sin embargo, estos cursos tienen muchas deficiencias en términos de actitudes, materiales pedagógicos y docentes. El trabajo explora las experiencias de los estudiantes de los grados $7 .^{\circ}$ y $8 .^{\circ}$ (edades entre 13 y 14) en los cursos de Ciudadanía y Educación en Derechos Humanos sobre la base de los datos cualitativos recogidos a través de discusiones de grupos focales en Ankara y Estambul en el año académico 2006-2007. Las respuestas de los estudiantes indican que estos cursos han tenido poco impacto en la capacitación de los estudiantes o en hacerles considerar sus propios u otros derechos humanos como parte integral de sus vidas. Por el contrario, los alumnos perciben los escenarios nacional y mundial como caracterizados por violaciones masivas de los derechos humanos contra los que se sienten impotentes. El documento llama la atención sobre la importancia de una revisión de la educación en derechos humanos para los estudiantes junto con un enfoque global y una metodología adecuada.

\section{África}

En África del sur existe un programa piloto que cubre los países de Mozambique, Namibia y Zimbawe. Rukanda (2002) centra su exposición en los problemas culturales que enfrenta la educación en derechos humanos. La autora sostiene que como la educación en derechos humanos trata más con el dominio afectivo, lo que se requiere es un cambio de actitud, a veces "poniendo el dedo en la llaga" de las costumbres y las tradiciones, y que los educadores en derechos humanos deben aceptar que tendrán que utilizar los métodos de formación y educación que ayudan a los estudiantes a enfrentar y cuestionar algunas de las creencias, actitudes y valores que habían dado por sentados y que pueden tener consecuencias negativas para los derechos humanos.

\section{América}

Para el caso latinoamericano en general es ilustrativa la obra de Magendzo (2005), en la que se narra el comienzo del 
trabajo con maestros en la educación en derechos humanos en América Latina a mediados de la década de los 80 , con los regímenes dictatoriales aún en el poder, y la comprensión de que el enfoque crítico era necesario en tales circunstancias. El autor refiere que el proceso de transformación de cambio no fue fácil, enfrentando muchos retos personales, sociales, políticos y culturales. En el artículo menciona algunos de esos desafíos, en particular:

a. La falta de un enfoque crítico.

b. La "incapacidad" cultural para reconocer al "otro" como un legítimo "otro" y aceptar la existencia de la diversidad social y cultural. Por último, el artículo da recomendaciones teóricas y prácticas sobre cómo superar estos dos obstáculos.

En el Caribe, Bajaj (2004) narra cómo un curso de derechos humanos de tres meses de duración, sobre la base de la investigación crítica fue ofrecido a estudiantes de octavo grado en un barrio marginal de Santo Domingo. Antes y después del curso se midieron actitudes, comportamientos y conocimientos de los principios de los derechos humanos de los estudiantes. El plan de estudios se centró en los principios internacionales y problemas locales arraigados, como la discriminación contra los inmigrantes haitianos, la brutalidad policial, la violencia contra las mujeres y la explotación del trabajo infantil en las zonas francas. En el trabajo se discute el campo de la enseñanza de los derechos humanos, los hallazgos sobre la naturaleza de la respuesta del estudiante frente al curso y su impacto en la identidad de los estudiantes, la solidaridad con las víctimas de abusos contra los derechos humanos y la confianza en sí mismos, como resultado de la educación en derechos humanos.

En la medida en que Canadá es un país reconocido por su alto cumplimiento y aplicación de los derechos humanos, es pertinente examinar el estudio liderado por Nazzari (Nazzari, McAdams, \& Roy, 2005), en el que examina las prácticas y condiciones esenciales para fomentar el aprendizaje transformativo mediante el programa de Formación en Derechos Humanos Internacionales de la Fundación Canadiense de Derechos Humanos. Se sugiere que los participantes del programa desafían sus propios valores y suposiciones acerca de los derechos humanos, su trabajo y su sociedad a través de la reflexión crítica. En consecuencia, 
sostienen que, si los educadores en derechos humanos contribuyen a la educación transformadora de los demás, es necesario que entiendan los fundamentos teóricos y prácticos del proceso de aprendizaje asociado con la educación en derechos humanos.

\section{Asia}

Un país asiático democrático (Japón) es estudiado por Takeda (2012). Señala que, aunque los derechos humanos a menudo se expresan como principios universales, el concepto fue concebido en un contexto sociopolítico e histórico particular. Las conceptualizaciones y práctica de los derechos humanos varían a través de las sociedades, y se enfrentan a numerosos retos. Después de proporcionar un recuento histórico de la conceptualización de los derechos humanos en la sociedad japonesa, el estudio examina la educación en derechos humanos en Japón, centrándose en la aplicación del Decenio de las Naciones Unidas para la Educación en Derechos Humanos. Mientras que el Plan de Acción del Decenio abogaba por un enfoque integral, la educación japonesa derechos humanos pone mucha menor atención en la transmisión de conocimientos y el desarrollo de actitudes de los alumnos, mientras hace un fuerte énfasis en los aspectos de la responsabilidad y de las relaciones humanas armoniosas entendidas en el contexto histórico de la educación moral japonesa. El texto contiene propuestas pedagógicas para promover un enfoque integral, que incluya atención a la función de empoderar a los alumnos, lo que les permita protegerse mediante la invocación de los derechos humanos

Mientras, en el sistema híbrido de China Leung (2008) ilustra la situación en Hong Kong, donde la HRE es considerada como un aspecto de la educación cívica. Durante décadas, la HRE fue descuidada, pero en 2009 se introdujo una nueva asignatura obligatoria para los estudiantes de secundaria superior (15-18 años): "Estudios Liberales". El artículo revisa el desarrollo de la educación en derechos humanos dentro de la educación cívica, seguido por el análisis de los documentos de Estudios Liberales con referencia a los documentos internacionales de derechos humanos. Se concluyó que los estudios liberales se pueden utilizar como un vehículo para la HRE. Los objetivos y la pedagogía propuestos son similares a las adoptadas por la HRE y las normas internacionales de derechos humanos se pueden incorporar en 
el contenido. Sin embargo, la educación en derechos humanos derivada de Estudios Liberales es una forma de "HRE pobre en acciones". Si esta "HRE pobre en acciones" puede cultivar adecuadamente una cultura universal de los derechos humanos se ve como un tema para futuras investigaciones.

El subcontinente indio está documentado en dos estudios. El primero, de la ya mencionada profesora Bajaj (2011), narra la historia de las iniciativas de educación en derechos humanos que han proliferado en las últimas tres décadas y que se pueden encontrar en las discusiones políticas, reformas de libros de texto y las iniciativas de base en todo el mundo. La autora explora específicamente el papel de los docentes en la HRE centrándose en un estudio de caso del programa de una ONG que opera en toda la India. Bajaj sostiene que la propia transformación de los docentes debe ser central en las discusiones sobre la reforma educativa y presenta los datos de una iniciativa de HRE gestionados por dicha ONG, argumentando que mientras los docentes de HRE pueden ser alentados a igualar las relaciones de poder dentro de las aulas y simultáneamente muchas comunidades semianalfabetas tienen a los docentes ( $y$ a los libros de texto) en alta estima, se sugiere que la defensa que los docentes hacen de los derechos humanos puede resultar fundamental para la educación en derechos humanos y llevarla más allá de los muros de la escuela, que es el objetivo último del proyecto educativo. Las conclusiones del estudio son:

a. La formación del profesorado que sea apropiado, contextualizado y atractivo incentiva la participación y legitima tanto el mensaje como los mensajeros de los derechos humanos.

b. La propia transformación y el interés por los derechos humanos de los docentes puede beneficiar a sus hogares, escuelas y comunidades de múltiples maneras.

c. Los docentes y los libros de texto pueden proporcionar legitimidad a los derechos humanos y ser los recursos vitales de la comunidad para intervenir en abusos de los que sean víctimas.

Por su parte, Khoja-Mooolji (2014) trabaja el caso de Pakistán y critica la adopción entusiasta de la HRE en contextos postcoloniales mediante la visibilización de los enredos políticos 
e ideológicos del discurso con los supuestos neoliberales de lo que es la ciudadanía. La autora usa la evidencia de un programa específico en HRE -una serie de campamentos de verano para niñas comprometidas, Mujeres Líderes del Mañana (WLT por sus siglas en inglés)- que ella implementó en Pakistán. Con metodología de la investigación narrativa examinó las clases de ciudadanos imaginados a través de su plan de estudios y las normas de liderazgo y comunidad promovidas por el mismo para argumentar que el programa puede ser interpretado como una tecnología del neoliberalismo en cuanto reproducía racionalidades neoliberales. Los individuos, sin embargo, no son simplemente objetos de saberes: ellos cooptan, resisten, negocian y se comprometen. La autora pasa a ser escéptica con su propia postura sobre desarrollo de WLT al reflexionar sobre los momentos de resistencia cuando los participantes no solo cuestionaron los supuestos del programa, sino que también se involucraron en dinámicas y produjeron nuevas mutaciones de la HRE. Este despliegue de un discurso globalizador en un entorno local permite hacer un llamado para la reconceptualización de la educación en derechos humanos múltiple, contingente y fluida en contextos postcoloniales.

\section{Conclusiones}

Los estudios expuestos en las páginas anteriores dan una idea global de los temas y tendencias internacionales contemporáneas en investigación sobre pedagogía de los derechos humanos y buscan ilustrar los puntos de partida y posibles metodologías que puedan tomar trabajos de investigación dentro de los programas académicos colombianos de posgrado que incorporan la temática de los DD. HH., DIH y DICA.

La pedagogía en derechos humanos no necesariamente pertenece al dominio de las ciencias jurídicas ya que se encuentran casos en que las matemáticas, la historia, la religión, la ciudadanía y los juegos de rol sirven como medio de difusión de las normas y valores de los derechos humanos.

La pedagogía de la enseñanza de los derechos humanos aún no ha consolidado métodos compartidos o comunes que permitan identificar estándares de formación. No obstante, es claro que todas las experiencias reseñadas implican la participación de los estudiantes en su propia formación y el abandono de métodos exclusivamente teóricos. 


\section{Referencias}

Al-Nakib, R. (2012). Human rights, education for democratic citizenship and international organizations: Findings from a Kuwaiti UNESCO ASPnet school. Cambrid Journal of Education, 42(1), 97-112. Recuperado de https://doi.org/10.1080/0 305764X.2011.652072

Andreopoulos, G. (2002). Human Rights Education and Training for Professionals. International Review of Education, 48(3-4), 239-249. Recuperado de https://doi. org/10.1023/A:1020326030923

Austin, W. C., McDowell, T., \& Sacko, D. H. (2006). Synergy Across the Curriculum: Simulating the Institution of Postwar Iraqi Government. Journal of Political Science Education, 2(1), 89-112. Recuperado de https://doi. org/10.1080/15512160500484127

Baccouche, T. (2002). L'expérience régionale arabe en matiére d'éducation aux droits de I'homme dans les situations d'apprentissage informel. International Review of Education, 48(3-4), 217-228. Recuperado de https://doi. org/10.1023/A:1020364131311

Bajaj, M. (2004). Human rights education and student self-conception in the DominicanRepublic. Journal of Peace Education, 1(1), 21-36. Recuperado de https://doi.org/10.1080/1740020032000178285

Bajaj, M. (2011). Teaching to transform, transforming to teach: exploring the role of teachers in human rights education in India. Educational Research, 53(2), 207221. Recuperado de https://doi.org/10.1080/00131881.2011.572369

Bengs, B. L., \& Augustin, B. J. (2010). Right Bombs, Right Target: Law of Armed Conflict Instruction for United States Air Force Officer Candidates. Journal of Applied Security Research, 5(3), 397-413. Recuperado de https://doi. org/10.1080/19361611003601231

Bowie, B. (2012). Human rights education and the post secular turn. Journal of Beliefs \& Values, 33(2), 195-205. Recuperado de https://doi.org/10.1080/13617672. 2012.694062

Burke, K. M. (2007). Human rights and the rights of the child, a panoramic view. Globalisation, Societies and Education, 5(3), 333-349. Recuperado de https://doi. org/10.1080/14767720701662048

Çayır, K., \& Bağli, M. T. (2011). 'No one respects them anyway': secondary school students' perceptions of human rights education in Turkey. Intercultural Education, 22(1), 1-14. Recuperado de https://doi.org/10.1080/14675986.2011.549641

Day, L. E., Vandiver, M., \& Janikowski, W. R. (2003). Teaching the ultimate crime: Genocide and international law in the criminal justice curriculum. Journal of Criminal Justice Education, 14(1), 119-131. Recuperado de https://doi. org/10.1080/10511250300085691

Druba, V. (2002). The Problem of Child Soldiers. International Review of Education, 48(3-4), 271-278. Recuperado de https://doi.org/10.1023/A:1020309527289

Gearon, L. (2008). Freedom from repression: some thoughts on totalitarianism for religious, spiritual and citizenship/human rights education. International Journal of Children's Spirituality, 13(1), 5-14. Recuperado de https://doi. org/10.1080/13644360701834791

Gundara, J. (2000). Religion, human rights and intercultural education. Intercultural Education, 11(2), 127-136. Recuperado de https://doi.org/10.1080/713665241

Gusenbauer, M. (2019, enero). Google Scholar to overshadow them all? Comparing the sizes of 12 academic search engines and bibliographic databases. Scientometrics, 118(1), 177-214.

Hart, S. N. (2002). Making Sure The Child's Voice Is Heard. International Review of Education, 48(3-4), 251-258. Recuperado de https://doi.org/10.1023/A:1020373914085

Hung, R. (2012). Being human or being a citizen? Rethinking human rights and citizenship education in the light of Agamben and Merleau-Ponty. Cambridge Journal of Education, 42(1), 37-51. Recuperado de https://doi.org/10.1080/03 05764X.2011.651202

Inagaki, A. (2002). Teaching Human Rights Education in Indonesian Schools. International Review of Education, 48(3-4), 279-280. Recuperado de https:// doi.org/10.1023/A: 1020365712268 
Jawoniyi, O. (2011). Children's rights and religious education in state-funded schools: an international human rights perspective. The International Journal of Human Rights, 16(2), 337-357. Recuperado de https://doi.org/10.1080/13642987.20 11.563512

Khoja-Moolji, S. (2014). Producing neoliberal citizens: critical reflections on human rights education in Pakistan. Gender and Education, 26(2), 103-118. Recuperado de https://doi.org/10.1080/09540253.2014.898025

Leung, Y. W. (2008). An 'action poor' human rights education: a critical review of the development of human rights education in the context of civic education in Hong Kong. Intercultural Education, 19(3), 231-242. Recuperado de https://doi. org/10.1080/14675980802078590

Liebel, M. (2002). Child Labour and the Contribution of Working Children's Organisations in the Third World. International Review of Education, 48(3-4), 265-270. Recuperado de https://doi.org/10.1023/A:1020328610451

Magendzo, A. (2005). Pedagogy of human rights education: a Latin American perspective. Intercultural Education, 16(2), 137-143. Recuperado de https://doi. org/10.1080/14675980500133549

Nazzari, V., McAdams, P., \& Roy, D. (2005). Using transformative learning as a model for human rights education: a case study of the Canadian Human Rights Foundation's International Human Rights Training Program. Intercultural Education,

16(2), 171-186. Recuperado de https://doi.org/10.1080/14675980500133614

Office of the High Commissioner for Human Rights (OHCHR). (2005-). Database on human rights education and training. Recuperado de https://hre.ohchr.org/ hret/intro.aspx Último acceso: 20 de noviembre de 2019

Parra Ávila, E. (2016). Temas y tendencias internacionales contemporáneas en investigación sobre pedagogía de los derechos humanos. Actualidades Pedagógicas, (67), 15-30. Recuperado de http://dx.doi.org/10.19052/ap.3454

Print, M., Ugarte, C., Naval, C., \& Mihr, A. (2008). Moral and human rights education: the contribution of the United Nations. Journal of Moral Education, 37(1), 115-132. Recuperado de https://doi.org/10.1080/03057240701803726

Reardon, B. A. (2002). Human Rights and the Global Campaign for Peace Education. International Review of Education, 48(3-4), 283-284. Recuperado de https:// doi.org/10.1023/A:1020369813176

Ross, J. I., \& Rothe, D. L. (2007). Swimming Upstream: Teaching State Crime to Students at American Universities. Journal of Criminal Justice Education, 18(3), 460-475. Recuperado de https://doi.org/10.1080/10511250701705446

Rukanda, M. (2002). Education for Human Rights Democracy in Southern Africa: A Pilot Project in Mozambique, Namibia and Zimbabwe. International Review of Education, 48(3-4), 285-286. Recuperado de https://doi.org/10.1023/A:1020321930015

Starkey, H. (2012). Human rights, cosmopolitanism and utopias: implications for citizenship education. Cambridge Journal of Education, 42(1), 21-35. Recuperado de https://doi.org/10.1080/0305764X.2011.651205

Takeda, S. (2012). Human rights education in Japan: an historical account, characteristics and suggestions for a better-balanced approach. Cambridge Journal of Education, 42(1), 83-96. Recuperado de https://doi.org/10.1080/0305764X.2011.651203

Vithal, R. (2005). Gender justice, human rights and measurement in the mathematics classroom. International Journal of Mathematical Education in Science and Technology, 36(8), 827-841. Recuperado de https://doi. org/10.1080/00207390500084914

Zembylas, M. (2013). The Teaching of Patriotism and Human Rights: An uneasy entanglement and the contribution of critical pedagogy. Educational Philosophy and Theory, 46(10), 1143-1159. Recuperado de https://doi.org/10.1080/00131857 .2013 .803238 